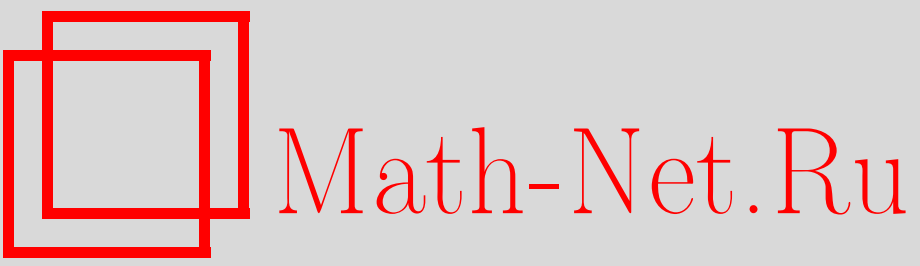

В. В. Абрамов, С. А. Бельман, Е. Ю. Лискина, Устойчивость по параметру при постоянно действующих возмущениях, Итоги науки и техн. Сер. Соврем. мат. и ее прил. Темат. обз., 2019, том 168, 9-14

DOI: https://doi.org/10.36535/0233-6723-2019-168-9-14

Использование Общероссийского математического портала Math-Net.Ru подразумевает, что вы прочитали и согласны с пользовательским соглашением

http://www.mathnet.ru/rus/agreement

Параметры загрузки:

IP : 54.198 .187 .58

26 апреля 2023 г., 18:25:10 


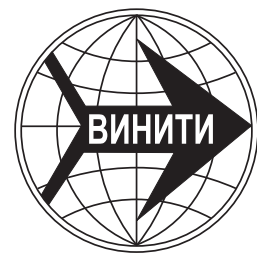

ИТОГИ НАУКИ И ТЕХНИКИ.

Современная математика и ее приложения.

Тематические обзоры.

Том 168 (2019). С. 9-14

DOI: $10.36535 / 0233-6723-2019-168-9-14$

УДК 517.925.51

\title{
УСТОЙЧИВОСТЬ ПО ПАРАМЕТРУ ПРИ ПОСТОЯННО ДЕЙСТВУЮЩИХ ВОЗМУЩЕНИЯХ
}

\author{
(c) 2019 г. $\quad$ В. В. АБРАМОВ, С. А. БЕЛЬМАН, Е. Ю. ЛИСКИНА
}

\begin{abstract}
АннотАция. Для нормальной периодической системы обыкновенных дифференциальных уравнений с малым параметром определено и исследовано свойство устойчивости начала координат. Предполагается, что правая часть системы имеет критическое линейное приближение. Условия устойчивости сформулированы в терминах оценок для оператора монодромии.
\end{abstract}

Ключевые слова: дифференциальное уравнение, малый параметр, устойчивость, постоянно действующее возмущение, оператор монодромии, периодическое решение.

\section{PARAMETER STABILITY UNDER CONSTANT PERTURBATIONS}

\author{
(c) 2019 V. V. ABRAMOV, S. A. BELMAN, E. YU. LISKINA
}

\begin{abstract}
For a normal periodic system of ordinary differential equations with a small parameter, we examine the stability property of the origin under the assumption that the right-hand side of the system has a critical linear approximation. The stability conditions are formulated in terms of estimates of the monodromy operator.
\end{abstract}

Keywords and phrases: differential equation, small parameter, stability, permanent perturbation, monodromy operator, periodic solution.

AMS Subject Classification: 34D20, 34C25

1. Введение. Свойство устойчивости начала координат при постоянно действующих возмущениях (ПДВ-устойчивость) имеет существенное приложение в теории управления. Классические достаточные признаки, заложившие традиции исследований по ПДВ-устойчивости, установлены прямым методом Ляпунова (см. [8,9]).

При определении ПДВ-устойчивости в правую часть системы вводится аддитивная добавка, из-за которой система может утратить нулевое решение. В определении используется оценка малости для добавки. Однако при этом не учитываются практически важные случаи согласованности возмущающей добавки с правой частью исследуемой системы. Такое согласование может реализоваться через зависимость исходной правой части и возмущающего воздействия от малого параметра. Поэтому свойство ПДВ-устойчивости целесообразно сформулировать и исследовать на основе понятия устойчивости по параметру (см. [11]). Для нормальной периодической системы обыкновенных дифференциальных уравнений ПДВ-устойчивость начала координат можно обнаружить на основе оценок степеней оператора монодромии (см. [5]). С этой целью используем подходы, примененные в $[1-4,6,7]$. В частности, для исследования можно применить следующую схему. Если при отклонении параметра от нулевого значения происходит ветвление малого периодического решения и это решение устойчиво по параметру, то при достаточно малом значении параметра и начальном значении любое решение остается в окрестности периодического решения, которое содержится в сколь угодно малой окрестности начала координат. 
Рассмотрим нормальную систему вида

$$
\dot{x}=g(t, x, \alpha),
$$

в которой $x \in \mathbb{R}^{n}, x \in I, I$ - неограниченный сверху промежуток, $\alpha \geqslant 0$ - малый вещественный параметр (случай, когда параметр числовой неотрицательный, возникает при наличии векторного параметра при выборе направления стремления параметра к критическому значению). Предположим, что система (1) удовлетворяет условиям существования и единственности решений $x\left(t, t_{0}, a, \alpha\right), x\left(t_{0}, t_{0}, a, \alpha\right)=a$ в окрестности точки $(a, \alpha)=\left(0_{n}, 0\right)$. Условие типа $g\left(t, 0_{n}, \alpha\right) \equiv 0_{n}$, при котором система (1) имеет нулевое решение, здесь не принципиально. Поэтому относительно точки $x=0_{n}$ в рассматриваемой системе при $\alpha>0$ можно предполагать наличие постоянно действующих возмущений.

Определение 1. Точка $x=0_{n}$ для системы (1) называется равномерно $\alpha$-устойчивой при постолнно действующих возмущениях (ПДВ-устойчива по параметру), если для любого $\varepsilon>0$ существуют числа $\delta_{1}>0, \delta_{2}>0$, для которых при всех $a \in \mathbb{R}^{n}, a \geqslant 0, t_{0} \in I$ из неравенств $\|a\| \leqslant \delta_{1}, \alpha \leqslant \delta_{2}$ следует, что при любом $t \geqslant t_{0}$ справедлива оценка

$$
\left\|x\left(t, t_{0}, a, \alpha\right)\right\|<\varepsilon .
$$

2. Вспомогательные результаты. Исследуем устойчивость по определению 1 для периодической по $t$ системы вида (1), когда $g(t \pm \omega, x, \alpha)=g(t, x, \alpha)$ при всех $(t, x, \alpha)$. При этом свойство устойчивости решения, сформулированное в терминах оценки нормы возмущения (устойчивость ляпуновского типа), имеет равномерный характер относительно начального момента $t_{0}$ (см. [9]). Поэтому без ограничения общности далее полагаем, что $t_{0}=0$. Будем также предполагать, что функция $g(t, x, \alpha)$ достаточно гладко зависит от фазовой переменной и параметра в окрестности точки $(x, \alpha)=\left(0_{n}, 0\right)$, а решения $x(t, a, \alpha), x(0, a, \alpha)=a$ системы локально продолжаемы на промежуток $t \in[0, \omega]$.

Лемма 1. Точка $x=0_{n}$ для $\omega$-периодической по $t$ системы (1) ПДВ-устойчива по параметру тогда и только тогда, когда для любого $\varepsilon>0$ существуют числа $\delta_{1}>0, \delta_{2}>0$, для которых из неравенств $\|a\|<\delta_{1}, 0 \leqslant \alpha<\delta_{2}$ следует, что при всех $s \in \mathbb{N}$ определено значение $x(s \omega, a, \alpha)$ и справедлива оценка $\|x(s \omega, a, \alpha)\|<\varepsilon$.

Доказательство. Необходимость данного утверждения очевидна, если в определении 1 взять $t=s \omega$. Достаточность установим по схеме доказательства [5, лемма 9.1]. В силу локальной продолжаемости решений числа $\delta$ и $\delta_{2}$ можно считать такими, что любое решение $x(t, x(s \omega, a, \alpha), \alpha)$ для всех $s \in \mathbb{N}$ определено при $t \in[0, \omega]$, если $\|a\|<\delta, \alpha<\delta_{2}$, т.е. продолжаемо вправо от $s \omega$. При этом любое решение $x(t, a, \alpha)$ нелокально продолжаемо по $t$. Произвольно зафиксируем $\varepsilon>0$. Выберем $\delta_{1} \leqslant \min \{\delta, \varepsilon\}$. В силу непрерывной зависимости решений системы $(1)$ от начальных значений и параметра можно считать, что при любых $\tau \in[0, \omega)$ из условий $\|a\|<\delta_{1}, \alpha<\delta_{2}$ следует оценка $\|x(\tau, a, \alpha)\|<\varepsilon$. Тогда по групповому свойству периодической динамической системы для произвольного $t=s \omega+\tau, s=[t / \omega]$, при всех $a$, удовлетворяющих условию $\|a\|<\delta_{1}$, и $\alpha$, удовлетворяющих условию $0 \leqslant \alpha<\delta_{2}$, получим оценку

$$
\|x(t, a, \alpha)\|=\|x(\tau,\|x(s \omega, a, \alpha)\|, \alpha)\|<\varepsilon .
$$

Итак, точка $x=0_{n}$ устойчива по определению 1 . Лемма 1 доказана.

Лемма 2. Если для любого $\delta_{1}>0$ существует такое $\delta_{2}>0$, что при $\|a\|<\delta_{1}, \alpha<\delta_{2}$ справедлива оченка $\|x(\omega, a, \alpha)\| \leqslant \delta_{1}$, то точка $x=0_{n}$ для $\omega$-периодической по $t$ системы (1) ПДВ-устойчива по параметру.

Доказательство. Произвольно зафиксируем $\varepsilon>0$. Выберем $\delta_{1}=\varepsilon$. По условию существует такое $\delta_{2}>0$, что при $\|a\|<\delta_{1}, \alpha<\delta_{2}$ справедлива оценка $\|x(\omega, a, \alpha)\| \leqslant \delta_{1}$. Допустим, для некоторого $s \in \mathbb{N}$ при $\|a\|<\delta_{1}, \alpha<\delta_{2}$ имеет место неравенство $\|x(s \omega, a, \alpha)\| \leqslant \varepsilon$. Тогда

$$
\|x((s+1) \omega, a, \alpha)\|=\|x(\omega, x(s \omega, a, \alpha), \alpha)\| \leqslant \varepsilon .
$$


Следовательно, по индукции $\|x(s \omega, a, \alpha)\| \leqslant \varepsilon$ при всех $s \in \mathbb{N}$, если $\|a\|<\delta_{1}, \alpha<\delta_{2}$. Итак, в силу леммы 1 точка $x=0_{n}$ устойчива по определению 1. Лемма 2 доказана.

Допустим, периодическая по $t$ система (1) имеет малое $\omega$-периодическое решение $x\left(t, a^{*}, \alpha\right)$, $a^{*}=a(\alpha) \neq 0_{n}, 0<\alpha<\Delta, \lim _{\alpha \rightarrow 0} a^{*}=0_{n}$.

Определение 2. [1]. Малое $\omega$-периодическое решение $x\left(t, a^{*}, \alpha\right)$ является $\alpha$-устойчивым, если для любого $\varepsilon>0$ существуют числа $\delta_{1}>0, \delta_{2}>0$, для которых из неравенств $\|u\|<\delta_{1}$, $0 \leqslant \alpha<\delta_{2}$ следует, что при всех $s \in \mathbb{N}$ определено значение $x\left(s \omega, a^{*}+u, \alpha\right)$ и справедлива оценка $\left\|x\left(s \omega, a^{*}+u, \alpha\right)-a^{*}\right\|<\varepsilon$.

Лемма 3. Если периодическая по $t$ система (1) имеет малое $\alpha$-устойчивое периодическое решение, то точка $x=0_{n}$ ПДВ-устойчива для системы (1).

Доказательство. Произвольно зафиксируем $\varepsilon>0$. Начальное значение произвольного решения $x(t, a, \alpha)$ системы (1) представим в виде $a=a^{*}+u$. Так как начальное значение малого периодического решения удовлетворяет условию $\lim _{\alpha \rightarrow 0} a^{*}=0_{n}$, то существует такое $\gamma>0$, что $\left\|a^{*}\right\|<\varepsilon / 2$ при $\alpha<\gamma$. По условию устойчивости периодического решения существуют $\gamma_{1}>0, \gamma_{2}>0$, для которых в силу неравенств $\|u\|<\gamma_{1}, 0 \leqslant \alpha<\gamma_{2}$ при всех $s \in \mathbb{N}$ определено значение $x\left(s \omega, a^{*}+u, \alpha\right)$ и справедлива оценка $\left\|x\left(s \omega, a^{*}+u, \alpha\right)-a^{*}\right\|<\varepsilon / 2$. Итак, при $\|a\| \leqslant\left\|a^{*}\right\|+\|u\|<\varepsilon / 2+\gamma_{1}=\delta_{1}$ и $\alpha<\delta_{2}=\min \left\{\gamma, \gamma_{1}\right\}$ имеет место неравенство

$$
\|x(s \omega, a, \alpha)\| \leqslant\left\|x\left(s \omega, a^{*}+\left(a-a^{*}\right), \alpha\right)-a^{*}\right\|+\left\|a^{*}\right\|<\frac{\varepsilon}{2}+\frac{\varepsilon}{2}=\varepsilon,
$$

т.е. точка $x=0_{n}$ ПДВ-устойчива для системы (1) по лемме 1 . Лемма 3 доказана.

3. Основные результаты. Найдем достаточные условия устойчивости по определению 1. С этой целью рассмотрим систему вида (1)

$$
\dot{x}=A(t) x+f(t, x, \alpha),
$$

в которой матрица $A(t)$ и вектор-функция $f(t, x, \alpha)$ являются $\omega$-периодическими по $t, f(t, x, \alpha)-$ локально достаточно гладкая относительно $x$ и $\alpha$ в окрестности точки $(x, a)=\left(0_{n}, 0\right)$, $\lim _{\alpha \rightarrow 0}\|f(t, \alpha x, \alpha)\| / \alpha \equiv 0$.

Заметим, что к системе вида (2) сводится система $\dot{y}=A(t) y+\alpha h(t)+\tilde{f}(t, x, \alpha)$ с периодической по $t$ правой частью, в которой $\lim _{\alpha \rightarrow 0}\|\tilde{f}(t, \alpha x, \alpha)\| / \alpha \equiv 0$, если система $\dot{z}=A(t) z+h(t)$ имеет периодическое решение $z(t)$. Для сведения достаточно сделать подстановку $y=x+\alpha z(t)$.

В силу леммы 2 при исследовании свойства устойчивости по определению 1 для системы (2) требуется локальная оценка оператора монодромии. Установим структуру этого оператора.

Пусть $X(t)$ - фундаментальная матрица системы $\dot{x}=A(t) x, X(0)=E, X=X(\omega)$ - матрица монодромии. В силу локальной гладкости правой части системы (2) устанавливается равенство

$$
X \int_{0}^{\omega} X^{-1}(\tau) f(\tau, X(\tau) a, \alpha) d \tau=p(a, \alpha)+\psi(a, \alpha),
$$

в котором $p(a, \alpha)$ - вектор-форма, $p(\alpha a, \alpha)=\alpha^{k} p(a, 1), k \in \mathbb{N}, k>1$. При этом функция

$$
\psi(a, \alpha)=X \int_{0}^{\omega} X^{-1}(\tau) f(\tau, x(\tau, a, \alpha), \alpha) d \tau-p(a, \alpha)
$$

удовлетворяет условию $\lim _{\alpha \rightarrow 0}\|\psi(\alpha a, \alpha)\| / \alpha^{k} \equiv 0$. Итак, для системы (2) оператор монодромии локально допускает выделение главной однородной части порядка $k$ относительно начального значения и параметра

$$
x(\omega, a, \alpha)=X a+p(a, \alpha)+\psi(a, \alpha) .
$$


В частности, это возможно, если в правой части системы $(2)$ функция $f(t, x, \alpha)$ локально допускает выделение главной вектор-формы $f(t, x, \alpha)=f_{k}(t, x, \alpha)+\tilde{f}(t, x, \alpha), f_{k}(t, \alpha x, \alpha)=\alpha^{k} f_{k}(t, x, 1)$, $\lim _{\alpha \rightarrow 0}\|\tilde{f}(t, \alpha x, \alpha)\| / \alpha^{k} \equiv 0$, которая не аннулируется при усреднении по решениям системы $\dot{x}=A(t) x$, т.е.

$$
p(a, \alpha)=X \int_{0}^{\omega} X^{-1}(\tau) f_{k}(\tau, X(\tau) a, \alpha) d \tau .
$$

Условия устойчивости по определению 1 для системы (2)-(3) сформулируем в терминах свойств функции $p(a, \alpha)$. При этом будем предполагать, что имеет место критический случай по линейному приближению: $\operatorname{det}(X-E)=0, \rho(X)=1(\rho(*)$ - операция вычисления спектрального радиуса матрицы).

Допустим, выделена матрица $P_{0}(a)$, удовлетворяющая условию $P_{0}(a) a=p(a, 0)$, причем существуют такие числа $c>0$ и $b>0$, что при всех $\lambda$, удовлетворяющих условию $\|\lambda\|=c$, и при всех малых $\gamma>0$ справедлива оценка

$$
\left\|X+\gamma P_{0}(\lambda)\right\| \leqslant 1-\gamma b .
$$

Рассмотрим систему (2)-(3) при $\alpha=0$. Согласно [5, лемма 9.2] задача об асимптотической устойчивости нулевого решения системы (2)-(3) сводится к задаче об асимптотической устойчивости нулевого решения системы в конечных разностях

$$
a_{j+1}=\left[X+P_{0}\left(a_{j}\right)+\Psi\left(a_{j}\right)\right] a_{j},
$$

где $\Psi(a)$-какая-либо матрица, удовлетворяющая условию $\Psi(a) a=\psi(a, 0)$. Так как $\lim _{\beta \rightarrow 0} \beta^{1-k}\|\Psi(\beta a)\|=0$, то существует такое $\delta_{1}>0$, что $\|\Psi(a)\|<b\|a\|^{k-1} / 2$ при всех таких $a$, что $\|a\|<\delta_{1}$. Тогда по условию (4) получим оценку

$$
\left\|a_{j+1}\right\|<\left(1-\frac{1}{2} b\left\|a_{j}\right\|^{k-1}\right)\left\|a_{j}\right\|<\left\|a_{j}\right\| .
$$

Выберем тождественную последовательность функций $V_{j}(u) \equiv\|u\|$. Для любого члена этой последовательности и для любых аргументов по свойствам нормы выполняются условия

$$
a(\|u\|)=\frac{\|u\|}{2} \leqslant V_{j}(u) \leqslant b(\|u\|)=2\|u\|, \quad\left|V_{j}(u)-V_{j}(\tilde{u})\right| \leqslant\|u-\tilde{u}\| .
$$

Кроме того, при всех малых $u$ справедливо неравенство

$$
\left\|u_{j+1}\right\|-\left\|u_{j}\right\|<-\frac{1}{2} b\left\|u_{j}\right\|^{k}<-c\left(\left\|u_{j+1}\right\|\right)=-\frac{1}{2} b\left\|u_{j+1}\right\|^{k} .
$$

Так как $a(\cdot), b(\cdot), c(\cdot)$ - функции класса Хана, то в силу [10, предложение 2] нулевое решение системы (5) асимптотически устойчиво.

Оценку (6) при $a=\beta \lambda, \beta>0,\|\lambda\|=,\|a\|<\delta_{1}$ представим в виде

$$
\|x(\omega, a, 0)\| \leqslant\left(1-\frac{\beta^{k-1} b}{2}\right)\|a\|<\delta_{1} .
$$

При этом существует такое малое $\delta_{2}>0$, что при $\alpha<\delta_{2}$ справедлива оценка $\|x(\omega, a, \alpha)\| \leqslant \delta_{1}$. Тогда по лемме 2 точка $x=0_{n}$ устойчива по определению 1.

Таким образом, установлено следующее утверждение.

Теорема 1. Если выполняется условие (4), то точка $x=0_{n}$ для системы (2)-(3) ПДВустойчива по параметру.

Заметим, что в силу асимптотической устойчивости нулевого решения системы (2)-(3) при нулевом значении параметра теорема 1 относится к некритическому случаю ПДВ-устойчивости.

Далее исследуем критические случаи устойчивости.

Допустим, функция $p(a, \alpha)$ в равенстве (3) удовлетворяет условию

$$
p\left(0_{n}, \alpha\right) \equiv 0_{n} .
$$


Кроме того, возможно разложение $p(a, \alpha)=P(a, \alpha) a$, при котором существует такое число $b>0$, что при всех $\lambda:\|\lambda\|=1$ и при всех малых $\gamma>0$ справедлива оценка

$$
\|X+\gamma P(\lambda, 1)\| \leqslant 1-\gamma b
$$

Теорема 2. Если выполняются условия (7) и (8), то точка $x=0_{n}$ для системы (2)-(3) ПДВустойчива по параметру.

Доказательство. В равенстве (3) выберем $a=\alpha \lambda,\|\lambda\|=1$. Выполним разложение

$$
p(a, \alpha)=\alpha^{k-1} P(\lambda, 1) a .
$$

Так как $\lim _{\alpha \rightarrow 0}\|\psi(\alpha \lambda \alpha)\| / \alpha^{k}=0$, то при некотором $\delta>0$ и при любом $\alpha<\delta$ имеет место неравенство $\|\psi(\alpha \lambda \alpha)\| \leqslant b \alpha^{k} / 2$. Тогда в силу условий (7) и (8) справедлива оценка

$$
\|x(\omega, a, \alpha)\| \leqslant\left\|X+\alpha^{k-1} P(\lambda, 1)\right\| \alpha+\|\psi(a, \alpha)\| \leqslant\left(1-b \alpha^{k-1}\right) \alpha+\frac{b \alpha^{k}}{2}=\left(1-\frac{b \alpha^{k-1}}{2}\right) \alpha<\alpha .
$$

Итак, для любого $\delta_{1} \leqslant \delta$ возьмем $\delta_{2}=\delta_{1}$ и получим, что при $\|a\|<\delta_{1}, \alpha<\delta_{2}$ справедлива оценка $\|x(\omega, a, \alpha)\| \leqslant \delta_{1}$, т.е. точка $x=0_{n}$ ПДВ-устойчива по параметру по определению 1 в силу леммы 2. Теорема 2 доказана.

Допустим, что существует число $b>0$, для которого при всех $\lambda$, удовлетворяющих условию $\|\lambda\|=1$, и малых $\gamma>0$ справедлива оценка

$$
\|\lambda+\gamma p(\lambda, 1)\| \leqslant 1-\gamma b .
$$

Теорема 3. Если выполняется условие (9), то точка $x=0_{n}$ для системы (1) ПДВ-устойчива по параметру.

Доказательство теоремы 3 аналогично доказательству теоремы 2.

Теорема 4. Пусть выполняются следующие условия:

(1) существует $a_{0} \in \operatorname{ker}[X-E]\left\{0_{n}\right\}$, при котором $p\left(a_{0}, 1\right)=0_{n}, \operatorname{rang}\left[p_{a}^{\prime}\left(a_{0}, 1\right) K p_{\mu}^{\prime}\left(a_{0}, 1\right)\right]=n$, $K-$-ундаментальная матрииа решений системы $[X-E] a=0_{n}$;

(2) существуют числа $c>0 u b>0$, для которых при всех $\lambda$, удовлетворяющих условию $\|\lambda\|=1$, м мальх $\alpha>0$ справедлива оценка $\|X+\alpha P(\lambda)\| \leqslant 1-a b$, в которой $P(\lambda)-$ матрича, удовлетворяющая условию $P(\lambda) \lambda=p\left(a_{0}+\lambda, 1\right)$.

Тогда точка $x=0_{n}$ для системы (1) ПДВ-устойчива по параметру.

По теореме 4 в системе (2)-(3) имеет место ветвление малого устойчивого по параметру периодического решения с направлением ветвления $a^{\prime}(0)=a_{0}$, которое устойчиво по определению 2 (см. [2]). Поэтому справедливость теоремы 4 следует из леммы 3.

\section{СПИСОК ЛИТЕРАТУРЫ}

1. Абрамов В. В. Двусторонняя устойчивость малого периодического решения// Вестн. РАЕН. - 2014. - 14, № 5. - C. 6-9.

2. Абрамов В. В. Ветвление периодического решения неавтономной системы с малым параметром// Вестн. РАЕН. - 2015. - 15, № 3. - С. 3-7.

3. Бельман C. А. Об условиях существования ненулевого периодического решения нелинейной системы дифференциальных уравнений с параметром// Изв. РАЕН. Диффер. уравн. - 2008. - № 13. - С. 5-16.

4. Бельман C. А. Периодические решения систем дифференциальных уравнений с параметром// Тр. Средневолж. мат. об-ва. - 2008. - 10, № 1. - С. 113-119.

5. Красносельский М. А. Оператор сдвига по траекториям дифференциальных уравнений. - М.: Наука, 1966.

6. Лискина Е. Ю. О периодических решениях системы дифференциальных уравнений// Изв. РАЕН. Диффер. уравн. - 2000. - № 3. - С. 60-65.

7. Лискина Е. Ю. Достаточные условия существования семейства периодических решений системы дифференциальных уравнений// Изв. РАЕН. Диффер. уравн. - 2001. - № 5. - С. 75-85.

8. Малкин И. Г. Теория устойчивости движения. - М.: Наука, 1966. 
9. Руш Н., Абетс П., Лалуа М. Прямой метод Ляпунова в теории устойчивости. - М.: Мир, 1980.

10. Халанай А., Векслер Д. Качественная теория импульсных систем. - М.: Мир, 1971.

11. Хапаев M. М. Асимптотические методы и устойчивость в теории нелинейных колебаний. - М.: Высшая школа, 1988.

Абрамов Владимир Викторович

Рязанский государственный университет имени С. А. Есенина

E-mail: v.abramov@365.rsu.edu.ru

Бельман Светлана Александровна

Рязанский государственный университет имени С. А. Есенина

E-mail: s.belman@365.rsu.edu.ru

Лискина Екатерина Юрьевна

Рязанский государственный университет имени С. А. Есенина

E-mail: e.liskina@365.rsu.edu.ru 